\title{
VI. On derivation of coexistence: Part I. Being the theory of simultaneous simple homogeneous equations
}

\section{J.J. Sylvester F.R.S. R.A.S.}

To cite this article: J.J. Sylvester F.R.S. R.A.S. (1840) VI. On derivation of coexistence: Part I. Being the theory of simultaneous simple homogeneous equations, Philosophical Magazine Series 3, 16:100, 37-43, DOI: 10.1080/14786444008649969

To link to this article: http://dx.doi.org/10.1080/14786444008649969

曲 Published online: 01 Jun 2009.

Submit your article to this journal $\pi$

Џll Article views: 4

Q View related articles $\square$ 
the purity of the nitric acid is a matter of indifference, strong commercial acid answering every purpose.

I remain, dear Sir, very truly yours, 82, Blackfriars Road, London, Dec. 10, 1839.

$$
\text { John Thomas Cooper. }
$$

P.S. I have found within these few days that some kinds of common coke, such as is very brilliant, close-grained, and has a columnar fracture, is equally good with the other varieties of carbon, and admits of being selected of almost any variety of form and size.

J. T. C.

VI. On Derivation of Coexistence : Part I. Being the Theory of simultaneous simple homogeneous Equations. By J. j. Syivester, F.R.S. \& R.A.S., Professor of Natural Philosophy in University College, London*.

Art. (1.) $W^{E}$ shall have constant occasion in this paper to affected with different subscribed numerical indices.

Such a letter is to be termed a " Base."

Every character consisting of a base and an inferior index I call an argument of the base, viz. the first, second, or $n$th argument, according as 1, 2, or in general $(n)$, be the number subscribed.

Art. (2.) I use the symbol PD to denote the product of the differences of the quantities to which it is prefixed (each being to be subtracted fiom each that follows); thus

$\mathrm{PD}(a b c)$ indicates $(b-a)(c-a)(c-b)$.

PD $(a \quad a b c)$ indicates $a b c(b-a)(c-a)(c-b$.

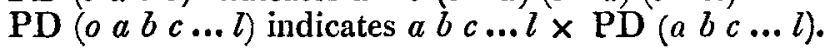

Art. (3.) For want of a better symbol I use the Greek letter $\zeta$ to denote that the product of factors to which it is prefixed is to be effected after a certain symbolical mannner. This I shall distinguish as the zeta-ic product.

The symbol $\zeta$ will never be prefixed except to factors, each of which is made up of one or more terms, consisting solely of linear arguments of different bases, i.e. characters bearing indices below but none above.

I am thereby enabled to give this short rule for zeta-ic multiplication: "Imagine all the inferior indices to become superior, so that each argument is transformed into a porwer of its base; multiply according to the rules of ordinary algebra; after the multiplication has been done fully out depress all the

* Communicated by the Author. Part I. appeared in L. \& E. Phil. Mag. Dec. 1839 , vol. xv. p. 428 . 
indices into their original position; the result is the zeta-ic product*."

Thus for example $\zeta\left(a_{r} \cdot b_{s}\right)$ is the same as simply $a_{r} \cdot b_{s}$, but $\zeta\left(a_{r}, a_{s}\right)$ represents not $a_{r}{ }^{\circ} a_{s}$ but $a_{r+s}$.

So in like manner

$$
\begin{aligned}
& \zeta\left(\left(a_{h}-b_{k}\right)\left(a_{l}-b_{m}\right)\right) \\
& \quad=a_{h+l}-a_{h} \cdot b_{m}-b_{k} \cdot a_{l}+b_{m+k} \\
& \begin{aligned}
\zeta\left(\left(a_{l}-b_{l}\right)\left(a_{l}-c_{l}\right)\left(b_{l}-c_{l}\right)\right) \\
\quad=\text { the depressed product of }(a-b)(a-c)(b-c) \\
\quad=\text { the depressed value of } a^{2}\left(b^{\prime}-c^{\prime}\right)+b^{2}\left(c^{\prime}-a^{\prime}\right)+c^{2}\left(a^{\prime}-b^{\prime}\right)
\end{aligned} \\
& \text { i.e. }=a_{2} \cdot b_{1}-a_{2} \cdot c_{1}+b_{2} \cdot c_{1}-b_{2} \cdot a_{1}+c_{2} \cdot a_{1}-c_{2} \cdot b_{1} .
\end{aligned}
$$

Art. (4.) We shall have occasion in this part to combine the two symbols $\zeta, \mathrm{PD}$ : thus we shall use

$\zeta \mathrm{PD}\left(a_{i}, b_{t}\right)$ to denote $\zeta\left(b_{1}-a_{i}\right)$

$\zeta \mathrm{PD}\left(a_{1} b_{1} c_{l}\right)$ to denote $\zeta\left(\left(b_{1}-a_{l}\right)\left(c_{1}-a_{l}\right)\left(c_{1}-b_{1}\right)\right)$.

Art. (5.) For the sake of elegance of diction I shall in future sometimes omit to insert the inferior index when it is unity; but the reader must always bear in mind that it is to be understood though not expressed.

I shall thus be able to speak of the zeta-ic product of such and such bases mentioned by name.

Art. (6.) We are not yet come to the limit of the powers of our notation. The zeta-ic product of the sum of arguments will consist of the sum of products of arguments, each argument being (as I have defined) made up of a base and an inferior index. Now we may imagine each index of every term of the zeta-ic product after it is fully expanded to be increased or diminished by unity, or each at the same time to be increased or diminished by 2 , or each in general to be increased or diminished by $r$. I shall denote this alteration by affixing an $(r)$ with the positive or negative sign to the $\zeta$. Thus $\zeta\left(a_{1}-b_{1}\right)\left(a_{1}-c_{1}\right)$ being equal to $a_{2}-a_{1} \cdot c_{1}+b_{1} \cdot c_{1}-b_{1} \cdot a_{1}$ $\zeta_{+1}\left(a_{1}-b_{1}\right)\left(a_{1}-c_{1}\right)$ is equal to $a_{3}-a_{2} \cdot c_{2}+b_{2} \cdot c_{2}-b_{2} \cdot a_{2}$ $\zeta_{-1}\left(a_{1}-b_{l}\right)\left(a_{i}-c_{l}\right)$ is equal to $a_{1}-a_{0} \cdot c_{0}+b_{0} \cdot c_{0}-b_{0} \cdot a_{0}$.

In like manner $\zeta \mathrm{PD}(a b c)$ indicating

$$
b_{2} \cdot a_{1}-b_{2} \cdot c_{1}+c_{2} \cdot b_{1}-c_{2} \cdot a_{1}+a_{2} \cdot c_{1}-a_{2} \cdot b_{1}
$$

* It is scarcely necessary to add that an analogous interpretation may be extended to any zeta-ic function whatever. Thus

$$
\begin{aligned}
& \zeta\left(a_{1}+b_{1}\right)^{2}=a_{2}+2 a_{1} b_{1}+b_{2} \\
& \zeta \cos \left(a_{1}\right)=1-\frac{a_{2}}{1.2}+\frac{a_{4}}{1.2 .3 .4}, \& c .
\end{aligned}
$$




$$
\begin{aligned}
& \zeta_{ \pm r} \mathrm{PD}(a \quad b c) \text { indicates } \\
& b_{2 \pm r} \cdot a_{1 \pm r}-b_{2 \pm r} \cdot c_{1 \pm r}+c_{2 \pm r} \cdot b_{2 \pm r}-c_{2 \pm r} \cdot c_{1 \pm r} \\
& +a_{2 \pm r} \cdot c_{1 \pm r}-a_{2 \pm r} \cdot b_{1 \pm \underline{r}} \text {. }
\end{aligned}
$$

I shall in general denote $\zeta_{+r} \overline{\mathrm{PD}}(a b c \ldots l)$ actually expanded as the zeta-ic product of $a, b, c, \ldots l$ in its $r$ th phase.

Art. (7.) General Properties of Zeta-ic Products of Differences.

If there be made one interchange in the order of the bases to which $\zeta$ is prefixed, the zeta-ic product, in whatever phase it be taken, remains unaltered in magnitude, but changes its sign.

Art. (8.) If in any phase of a zeta-ic product two of the bases be made to coincide, the expansion vanishes.

Art. (9.) Let $f_{1}$ be used, agreeably to the ordinary notation, to denote the sum of the quantities to which it is prefixed, $\int_{2}$ to denote the sum of the binary products, $\int_{3}$ of the ternary ones, and so on.

Thus let $\int_{1}\left(a_{1} b_{1} c_{l}\right)$ or $f_{1}(a b c)$ indicate $a_{1}+b_{1}+c_{1}$ and $f_{2}\left(a_{1} b_{1} c_{1}\right)$ or $f_{2}(a b c)$ indicate $a_{1} b_{1}+a_{1} c_{1}+b_{1} c_{1}$ and $f_{3}\left(a_{l} b_{l} c_{l}\right)$ or $f_{3}(a b c)$ indicate $a_{l} b_{1} c_{l}$

we shall be able now to state the following remarkable proposition connecting the several phases of certain the same zeta-ic products.

Art. (8.) Let $a, b, c, \ldots l$, denote any number of independent bases, say $(n-1)$; but let the arguments of each base be periodic, and the number of terms in each period the same for every base, namely $(n)$, so that

$$
\begin{array}{ll}
a_{r}=a_{r+n}=a_{r-n} & a_{n}=a_{0}=a_{-n} \\
b_{r}=b_{r+n}=b_{r-n} & b_{n}=b_{0}=b_{-n} \\
c_{r}=c_{r+n}=c_{r-n} & c_{n}=c_{o}=c_{-n} \\
\cdot \cdot \cdot \cdot \cdot \cdot \cdot & \cdot \cdot \cdot \cdot \cdot \\
l_{r}=l_{r+n}=l_{r-n} & l_{n}=\dot{l}_{0}=l_{-n}
\end{array}
$$

$r$ being any number whatever. Then

$$
\begin{aligned}
& \zeta_{-1} \mathrm{PD}(o a b c \ldots l)=\zeta\left(\int_{1}(a b c \ldots l) \cdot \zeta \mathrm{PD}(o a b c \ldots l)\right) \\
& \zeta_{-2} \mathrm{PD}(o a b c \ldots l)=\zeta\left(\int_{2}(a b c \ldots l) \cdot \zeta \mathrm{PD}(o a b c \ldots l)\right)
\end{aligned}
$$

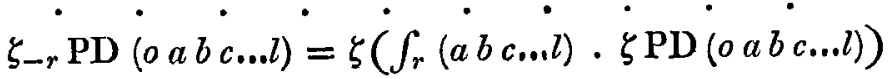


This proposition admits of a great generalization*, but we have now all that is requisite for enabling us to arrive at a proposition exhibiting under one coup d'ceil every combination and every effect of every combination that can possibly be made with any number of coexisting equations of the first degree, containing any number of repeated, or to use the ordinary language of analysts, (variable or) unknown quantities.

Art. (9.) For the sake of symmetry I make every equation homogeneous; so that to eliminate $n$ repeated terms, no more than $n$ equations will be required.

In like manner the problem of determining $n$ quantities from $n$ equations will be here represented by the case in which we have to determine the ratios of $(n+1)$ quantities from $n$ equations.

\section{Art. (9.) Statement of the Equations of Coexistence.}

Let there be any number of bases $(a b c \ldots l)$, and as many repeated terms $(x y z \ldots t)$, and let the number of equations be any whatever, say $(n)$. The system may be represented by the type equation

$$
a_{r} \cdot x+b_{r} \cdot y+c_{r} \cdot z+\cdot \cdot \cdot+l_{r} \cdot t=0 .
$$

In which $r$ can take up all integer values from $-\infty$ to $+\infty$. The specific number of equations given will be represented by making the arguments of each base periodic, so that

$$
a_{r}=a_{\mu n+r} b_{r}=b_{\mu n+r} c_{r}=c_{\mu n+r} . \quad \cdot l_{r}=l_{\mu n+r},
$$

$\mu$ being any integer whatever.

Art. (10.) Combination of the given Equations.Leading Theorem.

Take $f, g, \ldots k$ as the arbitrary bases of new and absolutely independent but periodic arguments, having the same index of periodicity $(n)$ as $a b c \ldots l$, and being in number $(n-1)$, i. e. one fewer than there are units in that index.

The number of differing arbitrary constants thus manufactured is $n \cdot(n-1)$.

Let $\mathbf{A} x+\mathbf{B} y+\mathbf{C} z+\ldots+\mathbf{L} t=0$ be the general prime derivative from the given equations, then we may make

$$
\begin{aligned}
& \mathrm{A}=\zeta \mathrm{PD}\left(\begin{array}{llll}
o & a f & \ldots & \ldots
\end{array}\right) \\
& \mathrm{B}=\zeta \mathrm{PD}(a b f g \ldots k) \\
& \mathrm{C}=\zeta \mathrm{PD}(o c f g \ldots k) \\
& \dot{\mathrm{L}}=\dot{\zeta} \mathrm{PD}(\text { o } l \dot{f} g \ldots \dot{k}) .
\end{aligned}
$$

Art. (11.) Cor. (1.) Inferences from the Leading Theorem. Let the number of equations, or, which is the same thing,

* See the Postscript to this paper for one specimen. 
the index of periodicity $(n)$, be the same as the number of repeated terms $(x y z \ldots t)$, then one relation exists between the coeflicients: this is found by making the $(n-1)$ new bases coincide with $(n-1)$ out of the old bases. We get accordingly, as the result of elimination,

$$
\zeta \mathrm{PD}(o a b c \ldots l)=0 \text {. }
$$

Art. (11.) Cor. (2.) Let the number of equations be one nore than that of the given bases, there will then be two equations of condition. These are represented by preserving one new arbitrary base, as $\lambda$. The result of elimination being in this case

$$
\zeta \operatorname{PD}(o a b c \ldots l \lambda)=0 .
$$

Ex. The result of eliminating between

is $\zeta \mathrm{PD}(o a b \lambda)=0$

$$
\begin{aligned}
& a_{1} \cdot x+b_{1} \cdot y=0 \\
& a_{2} \cdot x+b_{2} \cdot y=0 \\
& a_{3} \cdot x+b_{3} \cdot y=0
\end{aligned}
$$

i. e. $\lambda_{3} . b_{2} a_{1}-\lambda_{3} . b_{1} a_{2}+\lambda_{1} \cdot b_{3} a_{2}-\lambda_{1} \cdot b_{2} a_{3}+\lambda_{2} \cdot b_{1} a_{3}$

$$
-\lambda_{2} \cdot b_{3} a_{1}=0
$$

from which we infer, seeing that $\lambda_{3} \lambda_{2} \lambda_{1}$ are independent,

$$
\begin{aligned}
& b_{2} \cdot a_{1}-b_{1} \cdot a_{2}=0 \\
& b_{3} \cdot a_{2}-b_{2} \cdot a_{3}=0 \\
& b_{1} \cdot a_{3}-b_{3} \cdot a_{1}=0,
\end{aligned}
$$

any troo of which imply the third.

In like manner, in general, if the number of equations exceed in any manner the number of bases or repeated terms, the rule is to introduce so many nero and arbitrary bases as together with the old bases shall make up the number of equations, and then equate the zeta-ic product of the differences of zero, the old bases and the new bases, to nothing.

Art. (12.) Cor. (3). Let the number of equations be one fewer than the number $(n)$ of bases or repeated terms; the number of introduced bases in the general theorem is here $(n-2)$. Make these $(n-2)$ bases equal severally to the bases which in the type equation are affixed to $z, u \ldots t$, then

$$
\begin{gathered}
C=0 \\
D=0 \\
\cdots . . . . . . \\
\ddot{L}=0 . .
\end{gathered}
$$

and we have left simply

$$
\zeta \mathrm{PD}(o a c d \ldots k l) x+\zeta \mathrm{PD}(o b c d \ldots k l) y=0 \text {. }
$$

In like manner we may make to vanish all but $\mathrm{A}$ and $\mathrm{C}$, and thus get

$$
\zeta \mathrm{PD}(\text { o } a b d \ldots k l) x+\zeta \mathrm{PD}(o c b d \ldots k l) \approx=0,
$$


and similarly

$$
\begin{aligned}
& \zeta \mathrm{PD}(0 a b \ldots k) x+\zeta \mathrm{PD}(o b c \ldots l) t=0 .
\end{aligned}
$$

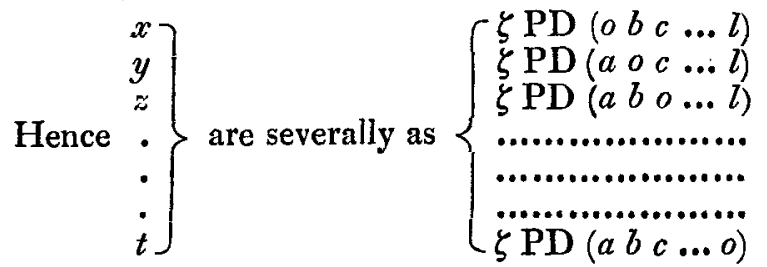

This is the symbolical representation as a formula of the remarkable method discovered by Cramer, perfected by $\mathrm{Be}-$ zout and demonstrated by Laplace for the solution of simultaneous simple equations.

Art. (13.) Cor. (4.) In like manner if the number of repeated terms be two greater than the number of equations, we have for the relation between any three of them, taken at pleasure, for instance, $x, y, z$,

$\zeta \mathrm{PD}(o a d \ldots l) x+\zeta \mathrm{PD}(o b d \ldots l) y+\zeta \mathrm{PD}(o c d \ldots l) z=0$.

And in like manner we may proceed, however much in excess the number of repeated terms (unknown quantities) is over the number of equations.

\section{Art. (14.) Subcorollary to Corollary (3.)}

If there be any number of bases $(a b c \ldots l)$, and any other two fewer in number $(f g \ldots k)$

$$
\begin{aligned}
& \zeta \mathrm{PD}(a f g \ldots k) \times \zeta \mathrm{PD}(b c \ldots l) \\
& +\zeta \mathrm{PD}(b f g \ldots k) \times \xi \mathrm{PD}(a c \ldots l) \\
& +\zeta \mathrm{PD}(a f g \ldots k) \times \zeta \mathrm{PD}(b c \ldots l) \\
& \dot{+} \dot{\mathrm{PD}}(\dot{i f g} \ldots \dot{b}) \times \dot{\zeta \mathrm{PD}}(a \dot{b} c . . .)=0,
\end{aligned}
$$

a formula that from its very nature suggests and proves a wide extension of itself.

In conclusion I feel myself bound to state that the principal substance of corollaries (i), (2) and (3) may be found in Garnier's Analyse Algébrique, in the chapter headed "Dévéloppement de la Théorie donnée par M. Laplace, \&c." But I am not aware of having been anticipated either in the fertile notation which serves to express them nor in the general theorems to which it has given birth.

$$
\text { End of Part (2). }
$$

[The subject to be continued.]

University College, London, Dec. 9, 1839.

P.S. I shall content myself for the present with barely enunciating a theorem, one of a class destined it seems to the 
Prof. Sylvester on Derivation of Coexistenee.

author to play no secondary part in the development of some of the most curious and interesting points of analysis.

Let there be $(n-1)$ bases $a, b, c \ldots l$, and let the arguments of each be " recurrents of the $n$th order*," that is to say let

$$
\begin{gathered}
a_{\iota}=\varphi\left(\cos \cdot \frac{2 \pi \iota}{n}\right) b_{\iota}=\psi\left(\cos \cdot \frac{2 \pi \iota}{n}\right) c_{\iota}=\chi\left(\cos \cdot \frac{2 \pi \iota}{n}\right) \\
\ldots \ldots l_{\iota}=\omega\left(\cos \cdot \frac{2 \pi \iota}{n}\right) .
\end{gathered}
$$

Let $R_{t}$ denote that any symmetrical function of the $r$ th degree is to be taken of the quantities in a parenthesis which come after it, and let $\checkmark$ indicate any function whatever. Then the zeta-ic product

$$
\zeta\left(\zeta \mathrm{R}_{t}(a b c \ldots l) \times \zeta_{\zeta} \vartheta \mathrm{PD}(o a b c \ldots l)\right)
$$

is equal to the product of the number

$$
\begin{aligned}
R_{t}( & \left(\cos \frac{2 \pi}{n}+\sqrt{-1} \cdot \sin \frac{2 \pi}{n}\right) \quad\left(\cos \frac{4 \pi}{n}+\sqrt{-1} \cdot \sin \frac{4 \pi}{n}\right) \\
& \left(\cos \frac{6 \pi}{n}+\sqrt{-1} \cdot \sin \frac{6 \pi}{n}\right) \cdots \cdots \\
& \left.\cos \left(\frac{(2 n-1) \pi}{n}+\sqrt{-1} \cdot \sin \frac{2(n-1) \pi}{n}\right)\right)
\end{aligned}
$$

multiplied by the zeta-ic phase

$$
\zeta_{\rho-t}, \vartheta \mathrm{PD}\left(\begin{array}{llllll}
0 & a & b & c & \ldots
\end{array}\right) !
$$

- I am indebted for this term to Professor De Morgan, whose pupil I may boast to have been. I have the sanction also of his authority, and that of another profound analyst, my colleague Mr. Graves, for the use of the arbitrary terms zeta-ic, zeta-ically. I take this opportunity of retracting the symbol S P D used in my last paper, the letter S having no meaning except for English readers. I substitute for it $Q D P$, where $Q$ represents the Latin word Quadratus. On some future occasion I shall enlarge upon a new method of notation, whereby the language of analysis may be rendered much more expressive, depending essentially upon the use of similar figures inserted within one another, and containing numbers or letters, according as quantities or operations are to be denoted. This system to be carried out would require special but very simple printing types to be founded for the purpose.

In the next part of this paper an easy and symmetrical mode will be given of representing any polynomial either in its developable or expanded form. 